\title{
The Expanding Role of Applications in the Development and Validation of CFD at NASA
}

\author{
David M. Schuster $^{1}$ \\ ${ }^{1}$ NASA Engineering and Safety Center, NASA Langley Research Center, Hampton, VA 23681, \\ USA
}

Keywords: CFD Application, method validation, flight data.

\begin{abstract}
This paper focuses on the recent escalation in application of CFD to manned and unmanned flight projects at NASA and the need to often apply these methods to problems for which little or no previous validation data directly applies. The paper discusses the evolution of NASA's CFD development from a strict "Develop, Validate, Apply" strategy to sometimes allowing for a "Develop, Apply, Validate" approach. The risks of this approach and some of its unforeseen benefits are discussed and tied to specific operational examples. There are distinct advantages for the CFD developer that is able to operate in this paradigm, and recommendations are provided for those inclined and willing to work in this environment.
\end{abstract}

\section{INTRODUCTION}

Traditionally, Computational Fluid Dynamics (CFD) methods developers have applied a very logical, systematic approach to software development, termed here as the "Develop, Validate, Apply" strategy. In this strategy, "Develop" refers to the coding and verification of the CFD software, while "Validate" refers to the process of running test cases on the software and comparing with known data. These validation data sources can be from other validated CFD methods, sub-scale experiments such as wind tunnel tests, or in rare cases, full-scale data, such as that obtained from flight tests. Upon completion of the validation phase of the strategy, the validated software system moves into the "Apply" stage and is delivered to the end-users who apply the method to problems that fit within the range of validation established for the method. This conservative strategy for CFD development ensures that the users of the method have a fully tested and validated method for their given application and as long as they don't stray too far from the parameters under which the method was validated, they have a tool that is capable of predicting flow characteristics within a demonstrated error band. However, as most anyone who develops CFD methods already knows, end-user applications can quickly migrate away from the parameters under which the software was validated.

In some cases, it becomes necessary for end-users to apply the methods to problems that are well outside the validation range of the software. Depending on the criticality of the pending analysis, the users may be forced into a situation where the final stages of the preferred strategy become switched and we find ourselves operating in the less systematic framework of
"Develop, Apply, Validate." This places the end-user in a situation where they have little or no knowledge of neither how the method will operate for their particular problem nor how accurate it will be. As a result, large modeling uncertainties are added to these types of analyses to account for this unknown performance. These additional uncertainties can have a profound impact on the prediction of performance and the overall design of a system. This paper discusses how one might be required to operate under this strategy and the ramifications of implementing this software maturation strategy.

\section{NASA'S EVOLVING CFD MATURATION PROCESS}

In the past seven years, the National Aeronautics and Space Administration (NASA) has seen a marked change in how they apply and ultimately develop and validate their Computational Fluid Dynamics (CFD) methods. This change comes as a result of two major Agency events. The first is the loss of the Space Shuttle Columbia during reentry in 2003. The second is the initiation of NASA's new exploration vision, embodied in the Agency's Constellation Program, which requires NASA engineer's to be responsible for large quantities of the aeroscience data products associated with the program's crewed spacecraft and launch vehicle. These two events coalesced to fundamentally change how the Agency applies its CFD methods, develops new CFD capability, and validates this capability.

\section{CFD and the Space Shuttle Program}

On January 16, 2003 NASA launched the Space Shuttle Columbia on what would turn out to be its $28^{\text {th }}$ 
and final mission. Roughly 82 seconds into the flight, a piece of insulating foam from the left bipod ramp on the vehicle's External Tank (ET) struck the left wing leading edge of Columbia, critically damaging the vehicle. Upon reentry, damage from the debris strike resulted in very high temperature flow reaching the vehicle's primary structure causing catastrophic structural failure and vehicle breakup over the Southwest United States. Following the accident, a formal investigation was conducted by a board of experts commissioned by the President of the United States. This Columbia Accident Investigation Board (CAIB) sponsored tests and analyses to determine the root cause of the accident and published their findings in a report commonly known as the CAIB Report ${ }^{1}$. Among the causes contributing to the accident, NASA's Organization and Safety Culture were identified as key contributors as "Shuttle Program management made erroneous assumptions about the robustness of a system based on prior success rather than on dependable engineering data and rigorous testing."

In partial response to the accident and the findings of the investigation, NASA established a new organization known as the NASA Engineering and Safety Center (NESC). The NESC operates under the philosophy of three primary tenets to ensure safety:

1) Strong in-line technical checks and balances to ensure proper engineering analysis and data are being applied to the problem.

2) Healthy tension between the Project, Safety, and Engineering components supporting the Program.

3) "Value added" independent assessment of problems that cannot be adequately resolved within the internal Program environment.

The NESC is funded through NASA's Office of the Chief Engineer, which allows it to maintain independence from the programs and projects it is asked to support. The independent assessment functionality of the NESC is one of the unique features of the organization that allows it to provide a technical evaluation of a given situation without biases due to schedule and budgetary pressures. The NESC presently supports 15 discipline-centric teams, each made up of experts from across NASA, other government agencies, industry, and academia. CFD is a key analysis tool of the Aerosciences Technical Discipline Team (TDT) as well as several other TDTs within the NESC.

The accident investigation resulting from the Columbia tragedy, as well as the highly increased technical insight into the Space Shuttle Program after it returned to flight status have stretched the Agency's CFD resources and capabilities. The fast-paced environment of the accident investigation required rapid turn-around of analyses that used our CFD methods on a scale that had not been anticipated during their development. This forced engineers to use the codes in innovative applications, sometimes with limited validation applicable to the specific problems they were analyzing. Since the accident, NASA has successfully flown the Space Shuttle 19 times through May, 2010, see Table 1, and the use of CFD to analyze and evaluate problems on the vehicle has continued to escalate and expand at a very high rate. Examples of CFD usage on the shuttle will be provided for the five bolded flights shown in the table.

Table 1. Space Shuttle Missions Since Columbia Accident

\begin{tabular}{|c|c|l|c|}
\hline Mission & $\begin{array}{c}\text { Launch } \\
\text { Date }\end{array}$ & Mission & $\begin{array}{c}\text { Launch } \\
\text { Date }\end{array}$ \\
\hline STS-114 & $\mathbf{7 / 2 6 / 2 0 0 5}$ & STS-126 & $\mathbf{1 1 / 1 4 / 2 0 0 8}$ \\
\hline STS-121 & $\mathbf{7 / 4 / 2 0 0 6}$ & STS-119 & $3 / 15 / 2009$ \\
\hline STS-115 & $9 / 9 / 2006$ & STS-125 & $5 / 11 / 2009$ \\
\hline STS-116 & $12 / 9 / 2006$ & STS-127 & $7 / 15 / 2009$ \\
\hline STS-117 & $6 / 8 / 2007$ & STS-128 & $8 / 28 / 2009$ \\
\hline STS-118 & $\mathbf{8 / 8 / 2 0 0 7}$ & STS-129 & $11 / 16 / 2009$ \\
\hline STS-120 & $10 / 23 / 2007$ & STS-130 & $2 / 8 / 2010$ \\
\hline STS-122 & $2 / 7 / 2008$ & STS-131 & $4 / 5 / 2010$ \\
\hline STS-123 & $3 / 15 / 2008$ & STS-132 & $5 / 14 / 2010$ \\
\hline STS-124 & $\mathbf{5 / 3 1 / 2 0 0 8}$ & & \\
\hline & & & \\
\hline
\end{tabular}

Far and away, the most dominant use of CFD on the shuttle since the Columbia accident has been for the prediction of launch debris and its transport. Prior to the Columbia accident, engineers and managers recognized the importance of predicting the transport of foam and ice debris during the ascent phase of the shuttle flight. Tools to predict debris transport were in development prior to the Columbia accident. Early debris prediction techniques used wind tunnel data to provide the aerodynamic environments necessary to make the predictions. In the late 1980's, CFD began to make inroads in predicting these environments ${ }^{2}$, albeit on simplified geometry configurations. By the mid-1990's, high-fidelity CFD analyses on much larger, more complex configurations were being used to predict shuttle aerodynamic environments ${ }^{3-4}$. These simulations matured quickly and generally produced accurate surface pressure predictions when compared with experimental data as shown in Figure $1^{5}$.

During this period debris trajectory prediction analyses were performed on a case-by-case basis and a high degree of automation of this process was not available. In addition, the aerodynamic characteristics of the debris itself were not simulated. The investigation of the Columbia accident necessitated the rapid development of an automated debris transport analysis capability. In addition, the CAIB also recommended that the complete debris environment encountered by the Space Shuttle Launch Vehicle during ascent be characterized prior to Return-to-Flight (RTF). Since detailed aerodynamic characteristics of the debris components were not included in previous 


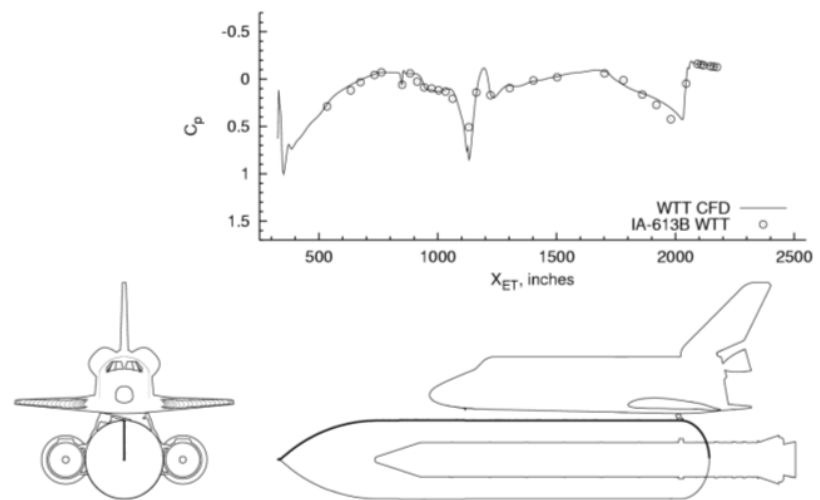

Figure 1. High-fidelity Space Shuttle CFD comparison with wind tunnel data (cf. Reference 5).

debris transport analyses, these characteristics had to be developed in parallel with the RTF analyses. Various debris shapes were characterized using CFD and these data were validated using ballistic range test results as shown in Figure $2^{6}$. Thus the engineers found themselves validating their methodology virtually in parallel with the analyses being used to prepare the vehicle for its return to flight. This started to blur the line between apply and validate in the traditional process of CFD method maturation. Once the Space Shuttle returned to operation, this line became increasingly transparent and in some cases, the validate/apply order became completely reversed.

\section{STS-114 - Space Shuttle Returns to Flight}

On July 26, 2005, over two years after the Columbia accident, NASA launched STS-114 marking the Space Shuttle's return to flight. Given the high sensitivity to debris and the uncertainty in many of the analyses employed leading up to this mission, numerous instruments and cameras were added to the flight manifest. In addition, maneuvers such as the "flip" maneuver of Figure 3, allowed the vehicle to be photographed and inspected to a level of detail unprecedented in prior flights. As a result of these inspections, a protruding gap filler was discovered between the ceramic tiles on the heatshield near the nose of the spacecraft. From the photographs, dimensions and shape of the protrusion could be derived and used as boundary conditions for the various boundary layer transition and heating analysis tools. These analyses, some involving CFD, were conducted over a span of just a few days while the vehicle was on orbit. The analyses showed the vehicle to be safe for return with the protruding gap filler, but uncertainties in the data produced sufficient concern for the safe return of the vehicle that mission managers formulated and exercised an Extra Vehicular Activity (EVA) to remove the offending component. Astronaut Steven Robinson was attached to the end of the arm of the Space Shuttle Orbiter's Remote Manipulator System and maneuvered to the front of the vehicle to remove the gap filler, as
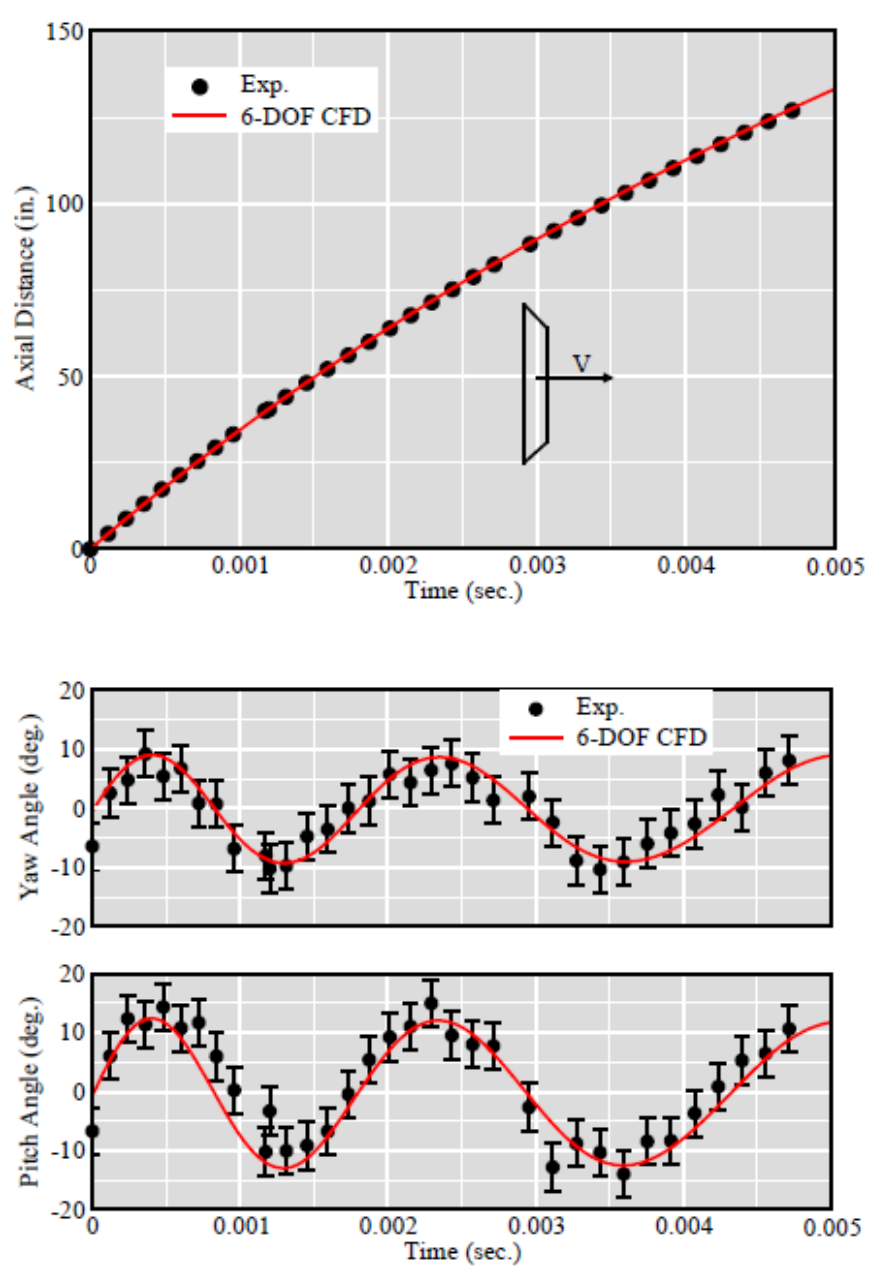

Figure 2. Comparison of aerodynamic characteristics of Space Shuttle debris computed by CFD with ballistic range data. (cf. Reference 6)

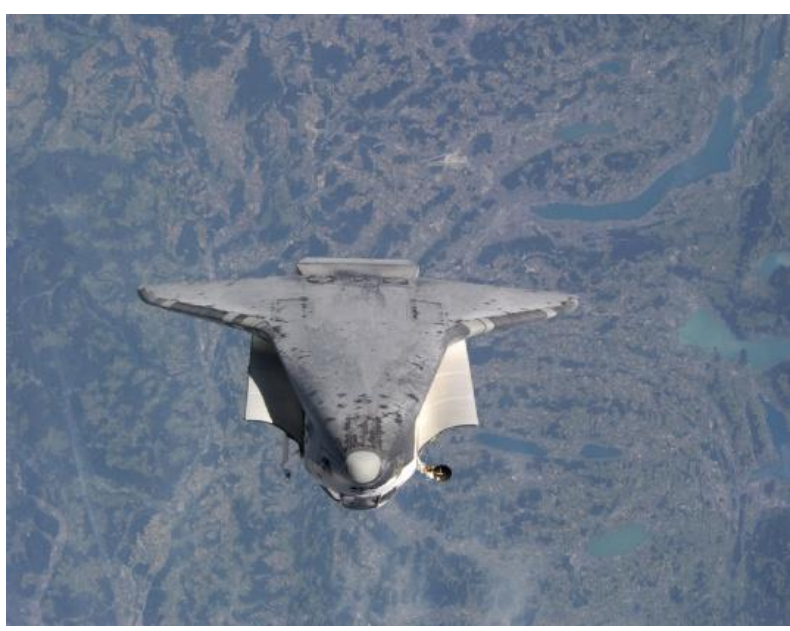

Figure 3. Space Shuttle performing flip maneuver as it approaches the International Space Station. 
seen in Figure 4. This high-risk EVA underscored the importance of accurate analysis capability to future missions and the impact it could have on the risk posture of these missions. The gap filler analysis was also a harbinger of how our analysis techniques would be stressed and extended during future flights.

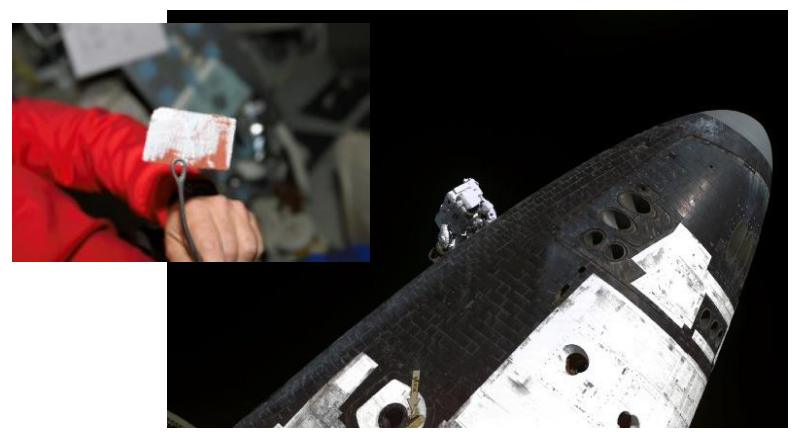

Figure 4. Removal of protruding heatshield gap filler during STS-114 mission.

During STS-114, numerous foam debris events were observed during the vehicle's ascent indicating that the foam debris issue was not as well understood as presumed prior to the flight. As a result, the program waited nearly a year to conduct its next flight. During this time, several large sources of foam debris were removed from the ET, including two large Protuberance Air Loads (PAL) Ramps. These ramps were part of the original ET design to protect externally mounted cable trays from high velocity crossflows generated during ascent. Considerable testing and analysis, including CFD, were performed prior to STS-114 to demonstrate that the cable trays remained structurally sound without the protection of the PAL ramps. The CFD involved unsteady separated flow over bluff bodies near a ground plane. This complex geometry and flow condition certainly stretched our CFD capability beyond the limits of its validation.

Qualification testing to demonstrate that the cable trays could withstand the aerodynamic loads of a space Shuttle launch included unsteady pressure and structural response data that could be used to partially validate the CFD analysis. But again these data were being acquired in parallel with the process being exercised to qualify the system for flight. Thus the validation and application of the CFD were being performed simultaneously.

The PAL ramps were identified as a potential debris source prior to STS-114, but the uncertainty of the analysis, testing, and the risk of unintended consequences resulting from removal of the PAL ramps outweighed the risk of a foam strike originating from one of the ramps. During the STS-114 mission though, a large portion of one of the PAL ramps was lost during ascent, Figure 5.

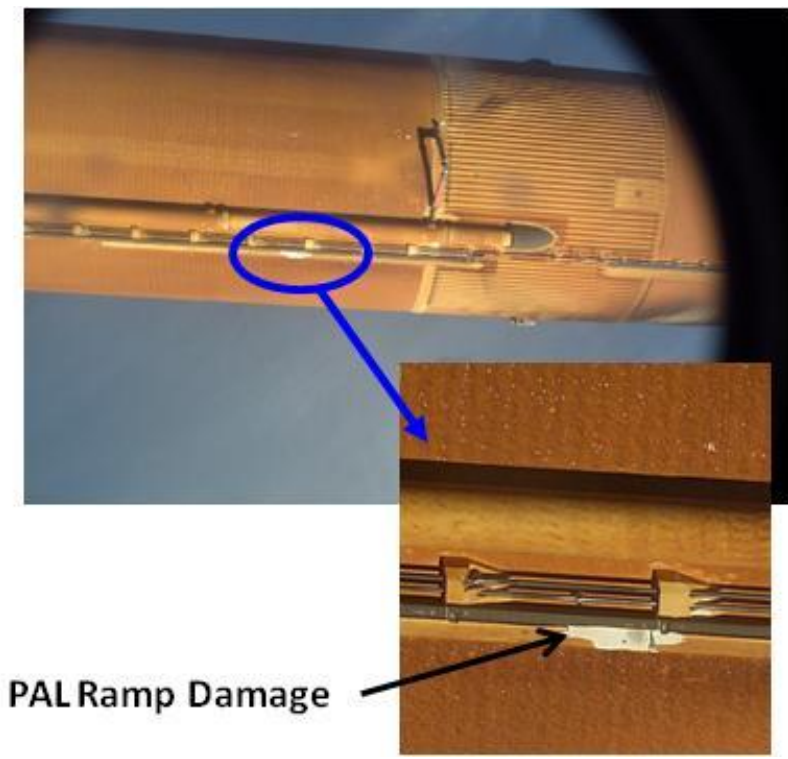

Figure 5. STS-114 PAL Ramp damage sustained during ascent.

This documented foam loss elevated the risk of the PAL ramps as a foam source, and they were subsequently removed from the ET for the second return to flight mission, STS-121, and subsequently for all future missions.

\section{STS-121 Returns Debris Transport Prediction Validation Data}

Due to the large volume of data acquired during STS-114 and the resulting analyses, testing, and system changes precipitated by this flight, STS-121 was launched nearly a year after STS-114. Imagery data acquired during STS-121 demonstrated that the number of foam liberation events had been significantly reduced and the mission proceeded with few flight issues. The relatively clean flight provided engineers with an opportunity to perform more detailed analyses of some of the data that had been collected on both STS-114 and STS-121. In particular, some of the debris transport methodology that had undergone such rapid development could finally be compared against flight data ${ }^{7}$. Figure 6 shows two images of foam debris captured during the STS-121 launch that was liberated from one of the Space Shuttle ET ice/frost ramps. The debris travels aft and passes near the starboard Solid Rocket Booster (SRB) nozzle. The video imagery, in this case from a camera mounted near the nose of the SRB, is of sufficient quality and resolution to roughly estimate the size and trajectory of the foam debris. Figure 7 shows a predicted trajectory for a piece of foam released from the same ice/frost ramp at this point in the ascent trajectory with the debris ultimately passing right over the top of the SRB as observed in flight. The ability to correlate computational methods against full-scale flight data in this manner is often very difficult to accomplish in the 


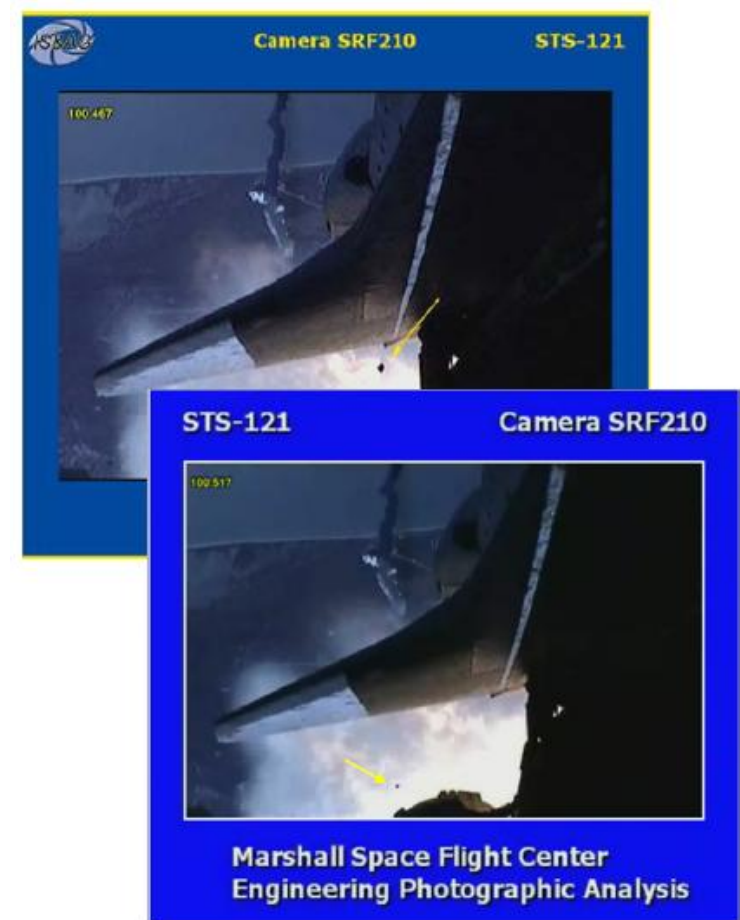

Figure 6. STS-121 ascent imagery of foam debris liberated from the Space Shuttle ET. (cf. Reference 7).

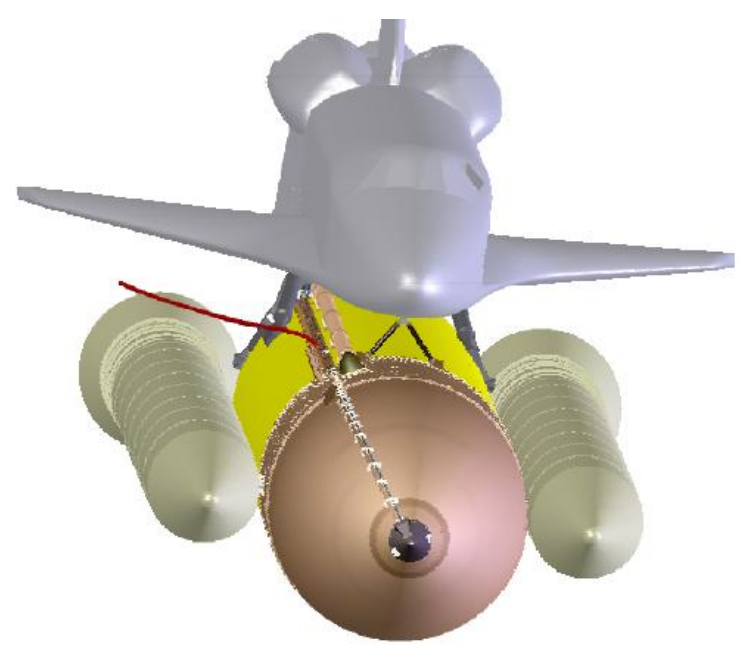

Figure 7. Predicted debris path (red line) for foam debris liberated from a specific ET ice frost ramp (cf. Reference 7).

standard "Develop, Validate, Apply" maturation strategy due to the cost of conducting a flight test solely for the purpose of method validation. However, when the application and validation tasks are performed in a more parallel fashion as with the foam debris trajectory prediction, full-scale validation opportunities are often more forthcoming, particularly if the method developers are tightly integrated into the application and the definition of the flight measurements that could be used for validation.

\section{STS-118 - Tile Damage Due to Debris}

During the post launch inspection of STS-118, significant heatshield tile damage was observed on a pair of tiles on the starboard wing as shown in Figure 8. Digital photographs taken from aboard the International Space Station (ISS) as the shuttle approached and performed its flip maneuver showed that the debris had dug into the tile enough to expose the underlying felt blanket that is used to mount the tiles to the vehicle's metallic structure. Thus, a small section of the vehicle was left without tile protection for atmospheric reentry. Measurements and geometry for the divot were estimated from the photographs and mathematical and physical models of the damage were constructed.

CFD was used to predict heating for the damaged area during entry, a sample of which is shown in Figure 9. The methods used to make these predictions had been validated for a variety of divot shapes, but this divot had a particularly deep, complex, two-tiered geometry, putting the heating tool predictions outside their

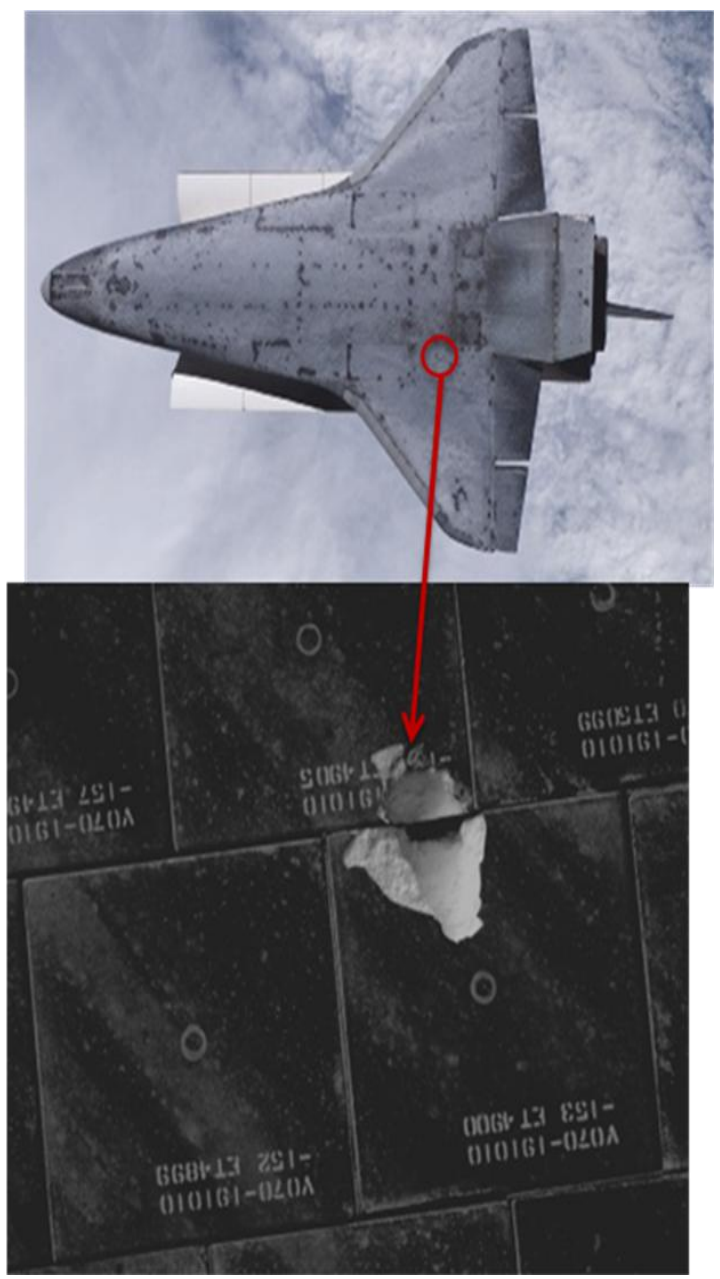

Figure 8. Tile damage incurred during the launch of STS-118. 


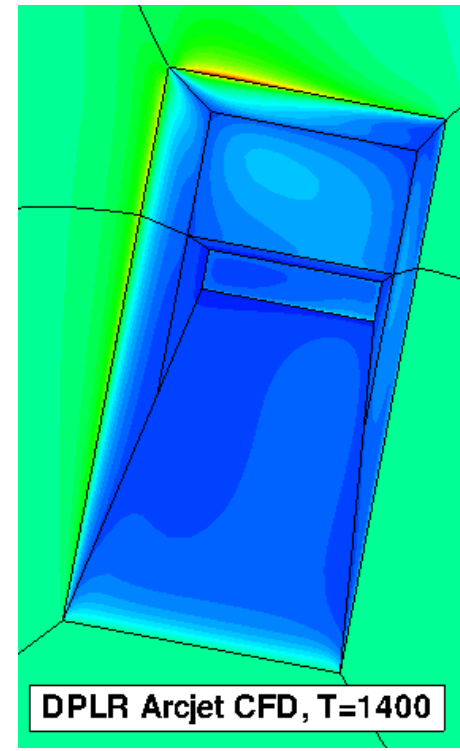

Figure 9. CFD prediction of heating due to STS-118 tile damage.

validation range. The heating tools predicted that the vehicle could reenter the atmosphere safely with the damage, but the uncertainty in the analysis required that further testing be performed in NASA's arc-jet test facilities before the vehicle could be cleared for entry. A model of the damaged tile system, shown in Figure 10, was constructed and quickly tested. Data from the heating tool were used to set the arc-jet flow conditions with significant margin beyond the heating levels predicted by the CFD. The conservative test conditions showed that the surrounding heatshield tiles would be damaged, but would not lose structural or thermal integrity. In addition, the exposed portion of the underlying metallic structure would not see temperatures high enough to damage the vehicles primary structure. The CFD analysis coupled with the conservative test data provided engineers and managers with the confidence to allow the vehicle to reenter the atmosphere without the need for a high-risk, highuncertainty repair. Ultimately the Space Shuttle Orbiter successfully returned to earth with little indication of damage due to excessive heating in this area.

All geometry modeling, model construction, analysis and testing for this effort were conducted in a matter of days while the vehicle was on orbit. Due to the complexity of the damage geometry, the heating tool used to predict the reentry temperatures at the damage site was used outside its validated range of applicability. But even though engineers and managers felt it necessary and prudent to conduct testing at conservative conditions to ensure the integrity of the thermal protection system, the CFD analysis played a significant role in the overall decision process to reenter the vehicle without repair. The method developers received some validation data on this geometry from the arc-jet testing prior to reentry, but in this case the application of the CFD had clearly begun to edge ahead of the method validation. Engineers and managers realized the value of the time critical data that could be extracted from the methodology even if it was applied beyond its validated range.

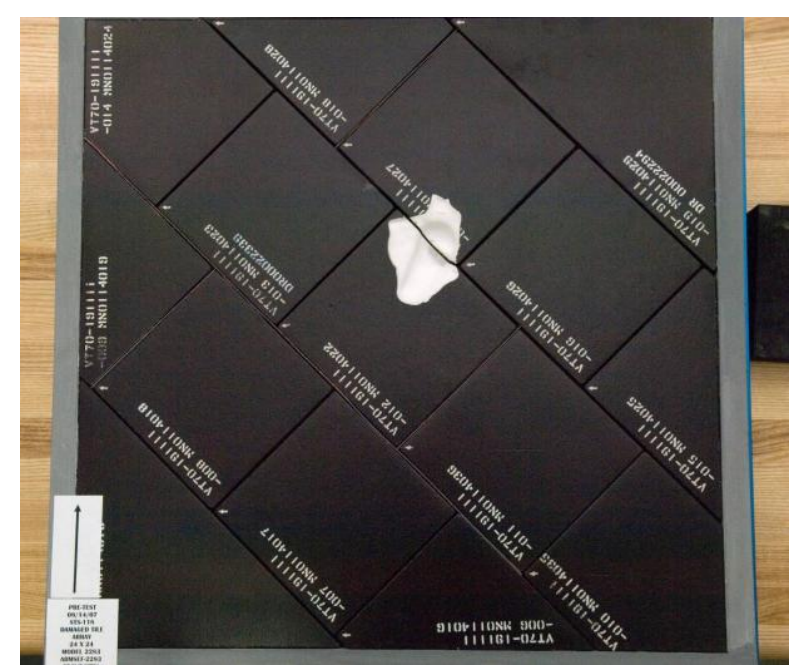

Figure 10. Model of STS-118 tile damage for use in arcjet testing.

\section{STS-124 - Launch Pad 39A Flame Trench Damage}

The launch of STS-124 caused significant damage to Kennedy Space Center's Launch Pad 39A. Shortly after SRB ignition, 3500 fire bricks were torn from the east wall of the Pad 39A SRB flame trench and exhausted north away from the launch pad, see Figure 11. Each of these bricks weighed approximately 20 $\mathrm{lbm}(9 \mathrm{Kg})$ and brick fragments were clocked using radar at up to $1000 \mathrm{ft} / \mathrm{sec}$ as they exited the flame trench. Beyond the damage to pad facilities, such as the flame trench, fencing, and other ground equipment, there was concern whether brick fragments, if liberated on future flights, could somehow make their way back to the Space Shuttle and damage it during its liftoff from the pad.

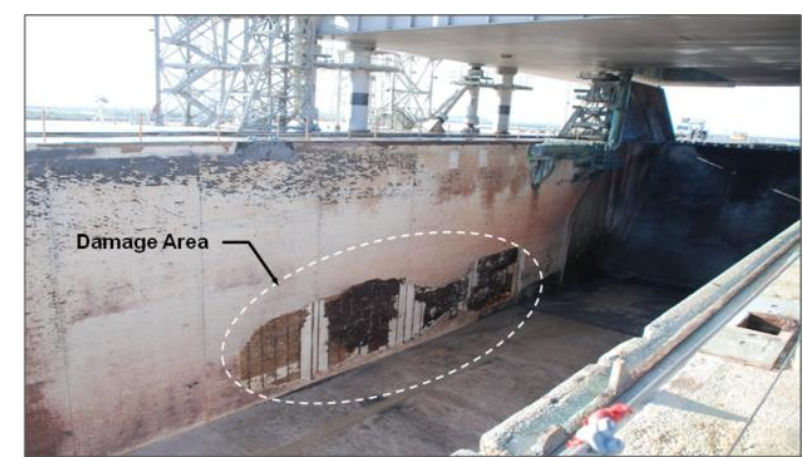

Figure 11. Kennedy Space Center Launch Pad 39A damage after launch of STS-124. 
CFD analysis was used to provide two critical sets of data in the subsequent investigation and for the flame trench repair. First, it was used to predict the time-dependent pressure and temperature environment along the walls of the flame trench as depicted in Figure 12. Second, it was used to predict the flowfield properties within the trench for use in debris transport analysis, as seen in Figure 13. This problem posed some extreme challenges for the CFD methods and those trying to apply them. First, the geometry is

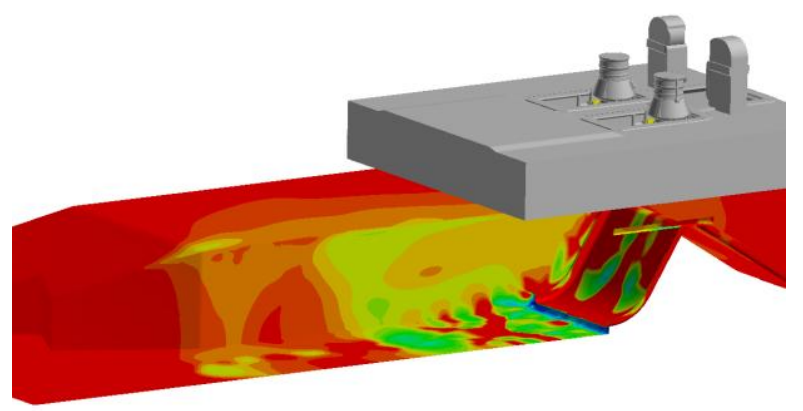

Figure 12. CFD prediction of the flow environment along the walls and floor of the Pad 39A flame trench shortly after SRB ignition.

very complex. The SRBs fire through a pair of holes in a part of the pad known as the Mobile Launch Platform (MLP). The MLP and the lower portion of the Space Shuttle SRBs are shown as the gray geometry in Figure 12. The two SRB plumes pass through the holes in the MLP and impact on the main exhaust flame deflector as depicted in Figure 13. The main flame deflector redirects the plume horizontally into the flame trench and allows it to exhaust out the north side of the pad and away from the launching vehicle. The other side of the main exhaust deflector redirects the three Space Shuttle Main Engine (SSME) plumes to the south and away from the vehicle.

In addition to the geometric complexity, there is significant flow physics complexity as well. The SRB plumes involve chemically reacting species exhausted at very high speeds and interacting with still air. The SRBs generate a severe ignition overpressure transient at startup that makes its way down the flame trench and is believed to have initiated the separation of the bricks from the east wall of the flame trench. Finally, the launch process involves the injection of an extremely large volume of water into the SRB holes and across the top surface of the MLP deck to provide acoustic suppression during the launch.

These physical and geometric features posed a severe challenge to the application of CFD, and forced its use well beyond the limits of validation and previous experience. The analysis was performed in a time accurate mode with two gases, one for the SRB plumes and the other for the surrounding air. Neither chemical reactions in the plumes or any attempt at simulating the water deluge were included in the analysis. The initial pressure and temperature transients predicted along the flame trench wall were used to provide structural design data used in the subsequent flame trench repair. The steady state flow data was used to feed debris transport predictions should a similar event occur in the future.

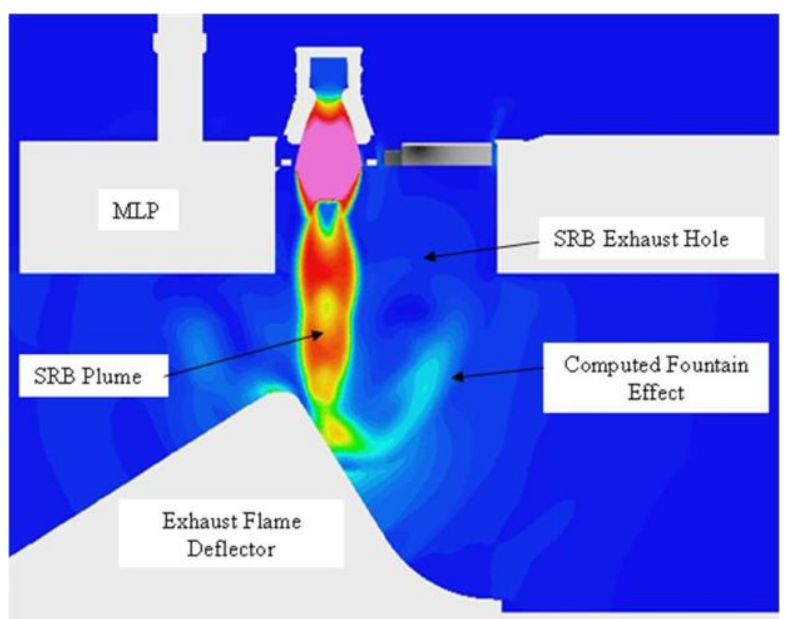

Figure 13. CFD prediction of SRB plume and associated flowfield during launch of STS-124.

The design for the flame trench repair was based on the CFD loads with significant margin included for modeling uncertainty. Similarly, the debris transport analysis involved significant deviations from the nominal predicted environments in the trench to ensure that a conservative estimate of the probability of debris striking the vehicle could be obtained. Again, this analysis and design was done on a very compressed schedule as repairs had to be accomplished prior to the next shuttle mission and the vehicle had to be cleared for debris impact before it could be launched. In the end, a repair could be formulated and applied to the launch pad on a schedule that did not impact the next launch and the debris transport analysis showed no credible mechanism for debris to impact the vehicle.

The significant point here is that the CFD analysis was knowingly applied well beyond its known range of applicability, and this was compensated for in the final design and debris analysis by adding large uncertainty margins to the predicted data. This approach had an unexpected beneficial aspect. The high uncertainties in the predictions provided motivation to acquire data for comparison with the flow predictions to help validate them and the designs and analyses they influenced. Instrumentation was added to the flame trench to measure key environments during the subsequent launch. Greater focus was placed on obtaining and analyzing debris transport information near the pad during subsequent launches as well. Thus, when engineers took the risk of applying their 
methods outside their comfortable range of validation and generated results with acknowledged high levels of uncertainty, they found that they could more effectively influence the acquisition of needed validation data for their application.

\section{STS-126 - Flow Control Valve Poppet Failure}

The final Space Shuttle example discussed in this paper is the failure of an Orbiter internal component that is used to control the pressure in the Liquid Hydrogen (LH2) propellant tank during launch. The LH2 tank, located in the ET is pressurized using a Gaseous Hydrogen (GH2) bleed from the SSMEs. Three Flow Control Valves (FCV) control the flow of $\mathrm{GH} 2$ back into the $\mathrm{LH} 2$ tank and thus manage the pressure in the tank. During the launch of STS-126, one of the FCVs transitioned to a high flow state without being commanded to do so and remained in that state for the remainder of the flight. Since the FCVs are located in the Space Shuttle Orbiter, they could be removed and inspected after the mission. This inspection revealed that a piece of the poppet on the suspect FCV had broken loose during the launch, as shown in Figure 14.

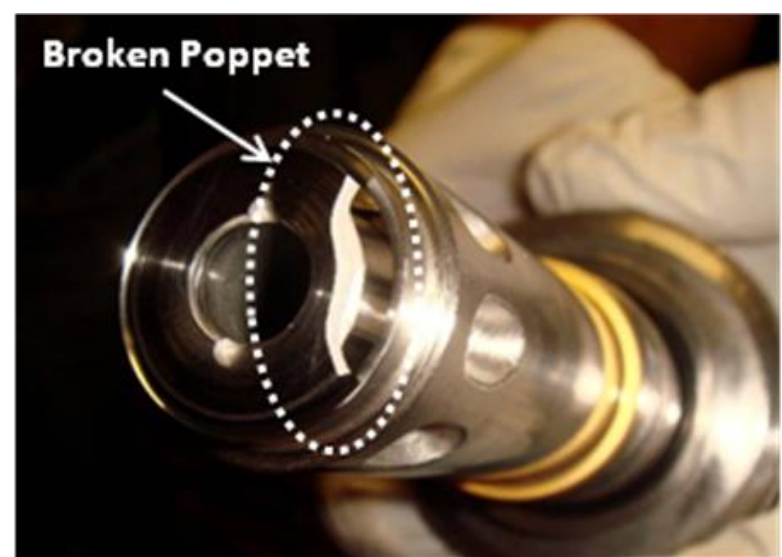

Figure 14. Inspection of SSME FCV after STS-124 mission reveals broken poppet.

The potential adverse consequences of this type of damage are twofold. First, with the broken poppet, the given FCV is free to pressurize the LH2 tank continuously and if the break is large enough or multiple FCV poppets simultaneously fail, the LH2 tank could be over-pressurized with catastrophic consequences. Second, if the energy and size of the poppet fragment is large enough, the fragment could be propelled downstream in the pressurization system with enough momentum to potentially puncture the $\mathrm{GH} 2$ repressurization piping as it tries to negotiate the various turns, bends, and diameter changes along the way from the Orbiter to the ET. This piping runs through areas in the orbiter, which if subjected to a gaseous hydrogen leak, could result in external combustion of the hydrogen, again with catastrophic results. In fact, borescope inspection of the pipes in the Orbiter downstream of the FCV showed several dents and scratches that were concluded to be caused by the failed poppet fragment as it made its way through the pipes. The fragment itself was never found and it was assumed to have been transmitted all the way to the ET where it was lost after stage separation.

Figure 15 shows a cutaway schematic of a FCV with the $\mathrm{GH} 2$ flow path indicated. $\mathrm{GH} 2$ enters the system from the top at very high pressure and flows around the poppet, which is translated left and right in the figure to open and close the flow path. The GH2 then enters the outlet tube and flows though a long system of pipes to the LH2 tank in the ET. The poppet position is controlled by a solenoid in the FCV. When the subject poppet fragment broke off, it created an open flow path for $\mathrm{GH} 2$ that could not be shut off.

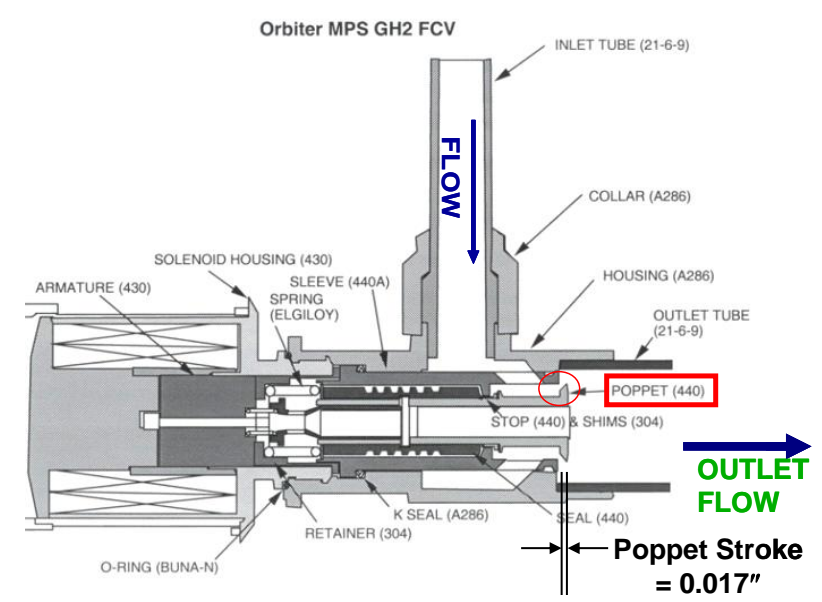

Figure 15.FCV schematic showing GH2 flow path and poppet control valve.

CFD and debris transport analysis were again key contributors to the investigation of this problem and the risk analysis for future flights that might encounter a similar issue. The FCV flow path, including the flow through much of the downstream piping was modeled using CFD. Both nominal and broken poppet configurations were analyzed and steady state and transient analyses were performed. Figure 16 shows a two-dimensional steady state CFD analysis of the flow through a nominal FCV with an intact poppet. The stream traces show the complex nature of the predicted flowfield downstream of the poppet. There is a very high pressure ratio between the flow upstream of the poppet and that downstream resulting in a very strong jet that emerges from the poppet and impacts the wall

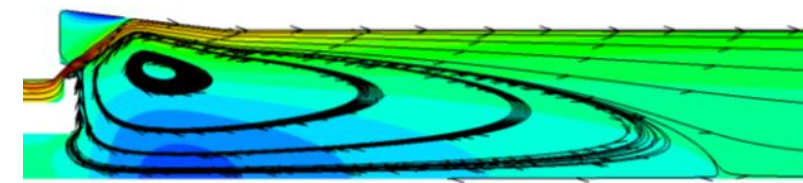

Figure 16. Two-dimensional steady CFD analysis of the nominal FCV operation. 
of the outlet tube. Two regions of recirculating flow are generated by this jet, a clearly visible, large region below the jet and directly behind the poppet and a smaller area in the triangular region above the jet.

Figure 17 shows a transient analysis of the developing flowfield behind a broken poppet just after it has fragmented. In this case, the poppet was fully closed and there was low flow mass through the poppet when it broke. In this case, classical one-dimensional flow features such as the propagating shock and contact discontinuity can be identified in the more complex two-dimensional flow. This type of information was used to estimate the downstream acceleration of the poppet fragment immediately after it was released from the poppet. These data were then input to a modified version of the debris transport methodology to predict the trajectory and velocity of the fragment as it moved downstream.

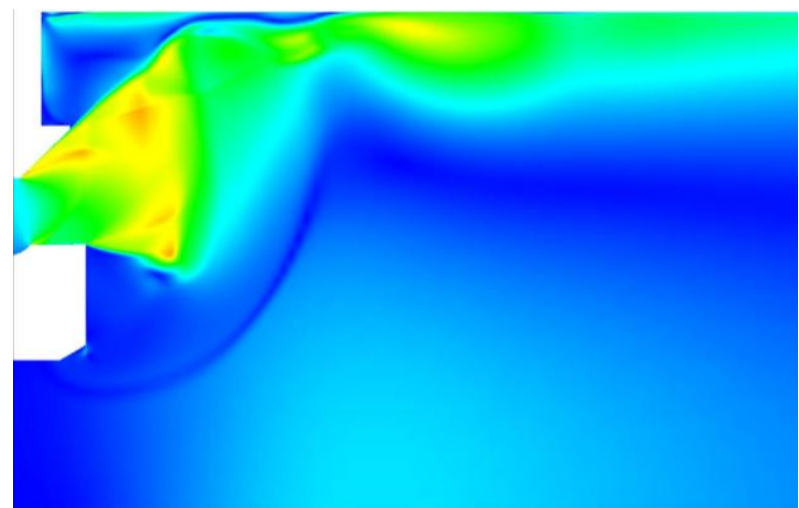

Figure 17. Transient CFD analysis of FCV flow just after poppet has broken.

This analysis required that not only the CFD be stretched well beyond its validated range of application, but also the debris transport methodology used to predict the trajectory of the poppet. In fact, new debris transport capability for pipe flows with multiple impacts was developed on the fly as the analysis progressed. Some testing was conducted in parallel with the debris trajectory application and development, but this testing was very cursory in nature. The debris simulations were used to perform a Probability Risk Assessment (PRA) for poppet debris released from a FCV From the PRA, guidelines were established for the maximum size of poppet debris that could be tolerated without sustaining collateral damage to the downstream pipes. Ultimately the problem was solved by requiring highly detailed inspection of the poppets prior to each flight, paying particular attention to poppets that have been used a high number of times.

\section{Space Shuttle Lessons Learned}

During the Columbia accident investigation, engineers found that the CFD codes provided data that appeared to be physically realistic, but they often only had limited test data to quantitatively verify their answers. Ultimately, many of the analyses performed during the accident investigation showed sufficient promise that significant efforts were undertaken to improve tools in anticipation of broader support of future flight operations. This provided the developers of these tools an opportunity to take a step back and formulate experiments and strategies to further and more rigorously validate and calibrate the new tools. However, the increased technical insight into postColumbia Space Shuttle flights only generated a broader range and greater diversity of problems that required innovative use of our CFD methods. In addition, these applications were all performed on a time-critical basis since they required answers to be generated while the vehicle was on orbit, or in the days and weeks leading up to a launch.

NASA engineers sometimes found themselves employing their CFD methods to previously unanticipated applications with minimal quantitative data against which to measure their results. If a given on-orbit or preflight problem generated a long-term requirement for a future similar capability, then testing could be formulated and conducted after the fact to help validate the new capability and bring it into the stable of tools used to support future flights. If the problem was deemed to be a one-of-a-kind application with little or no requirement for future application, then no data to support the application might ever be generated. Regardless of its future applicability, the CFD analyses of these time-critical problems were often the only data that could be generated on a timescale necessary to address the problem and with sufficient accuracy to characterize the issue. Thus NASA engineers and researchers sometimes found themselves shifting into the "Develop, Apply, Validate" paradigm. From a technical rigor standpoint this is a less than ideal approach to developing new capability, but interestingly, the approach has opened doors for developers to acquire data that generally is extremely difficult to obtain in the more ideal "Develop, Validate, Apply" maturation strategy; namely full-scale operational and flight data.

With the CFD techniques being applied in this increasingly aggressive fashion, engineers were forced to add generous uncertainty margins to their results to account for the fact that they didn't have sufficient data or experience against which to benchmark their analyses. It became commonplace to see $20 \%$ to $30 \%$ additional margin added to computational results as a "modeling uncertainty factor." Obviously adding this type of margin has operational and vehicle performance implications. As a result, Project Managers often become more motivated to acquire data to reduce these uncertainty margins. Often this data comes from the flight system it most directly impacts through additional instruments and data systems on the flight vehicle. Flight data has always been the gold standard 
for validation of CFD methods, but acquisition of this type of data is typically difficult, if not impossible, to obtain in a focused, standalone CFD development effort. Thus the aggressive application of NASA's CFD methods within the Space Shuttle Program has led to an increased number of opportunities to obtain highly sought after flight data for method evaluation and validation; a turn of events that certainly could not have been envisioned a priori.

\section{NASA's Constellation Program Further Reinforces the Paradigm Shift}

This evolutionary process has been continued with NASA's efforts to provide a broad array of aerodynamic and aerothermodynamic data products in support of the Agency's Constellation Program (CxP) manned spaceflight initiative. CxP designs have pressed engineers to predict the performance of aerodynamically complex vehicles, again with minimal applicable validation data against which to benchmark their methods. The CxP vehicles require analysis of a broad spectrum of flow issues, ranging from highly separated, bluff body flows to multiple plume jet interaction flows. Speed ranges run the gamut from the very low velocities of launch pad winds through transonic flight during vehicle ascent to hypersonic reentering flight.

The CxP has focused its early efforts on the development of two primary components, the Orion spacecraft and the Ares I launch vehicle. The Ares I, depicted in Figure 18, consists of a Space Shuttle heritage solid rocket booster as a first stage and a larger diameter liquid rocket second stage. From a CFD standpoint, major challenges posed by the Ares I include unsteady separated flows generated by the diameter change in the first/second stage transition and protuberances located at numerous locations on the vehicle. These unsteady separated flows are particularly important for definition of aeroacoustic and buffet environments for structural loads. There are also a number of smaller reaction control motors incorporated on the vehicle that perform tasks like vehicle roll control and stage separation. Since the SRB first stage is design to be recovered and refurbished for flight, similar to the Space Shuttle, it must tumble after stage separation. Otherwise, if it were to trim nose or tail first, the recovery parachutes would likely fail due to high opening loads. Therefore, by definition, the tumbling flight of the separated first stage involves significant unsteady separated flow.

Finally, the Ares I is a very high length to diameter ratio vehicle. This presents significant modeling problems given the small details, such as protuberances, that are important to the vehicle performance and must be modeled with sufficient resolution to capture their induced aerodynamics. As a result, the Ares I grid models are extremely large and costly to analyze.

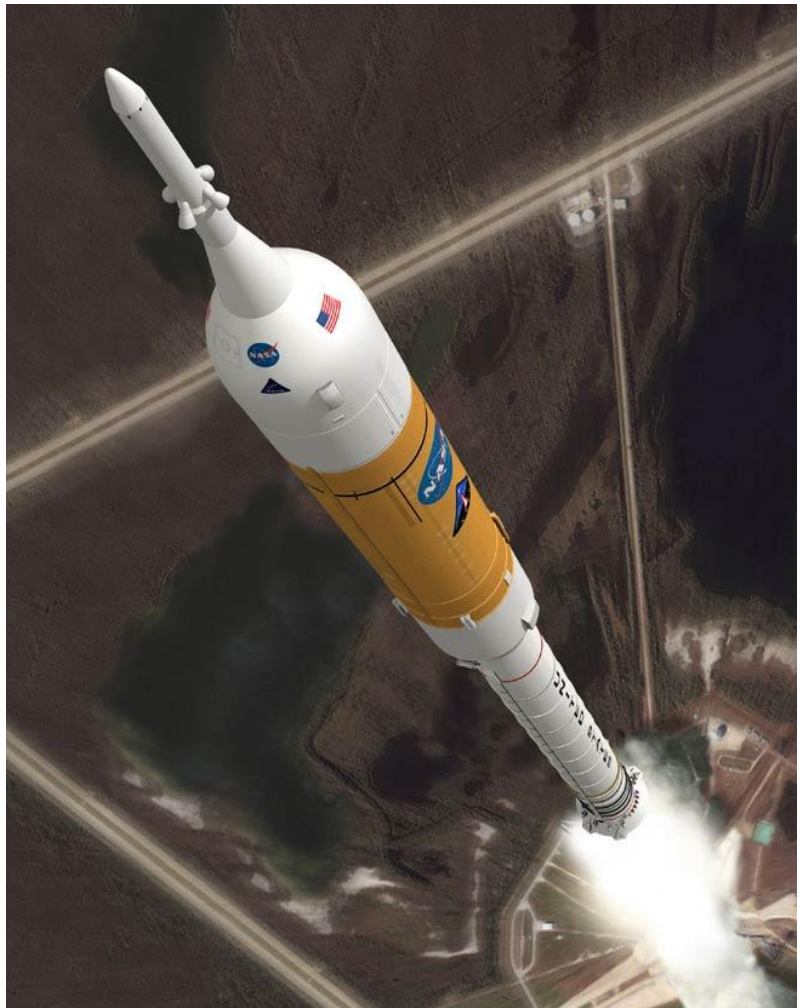

Figure 18. Artist concept of the Ares I launch vehicle.

Ground wind loads are also important to the vehicle as it rolls out and sits on the launch pad prior to flight.

By outward appearance, the Orion spacecraft, shown in Figure 19, is similar in shape to NASA's Apollo spacecraft, but it is much larger and heavier.

Aerothermodynamic issues during reentry dominate the CFD analysis of this vehicle. At present, the majority of design effort is being spent on earth entry from Low Earth Orbit (LEO), as in a mission to the ISS.

However, the vehicle is also expected to operate beyond LEO, where earth entry will be at much higher enthalpy. This brings issues for which CFD is not highly developed into the analysis space, such as radiation effects. Reaction Control System (RCS) jet interaction with the surrounding vehicle aerodynamics is also important for this vehicle. CFD is not well

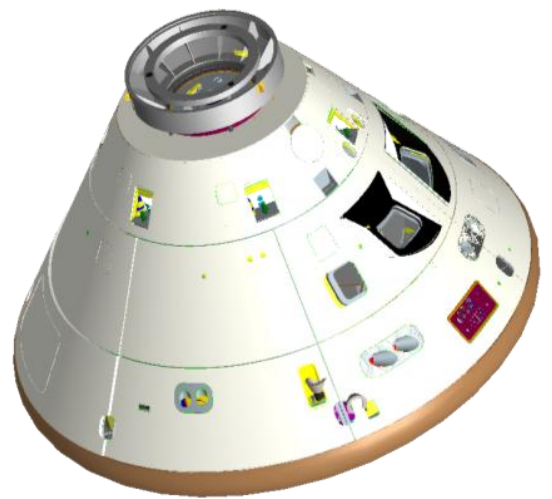

Figure 19. NASA’s Orion spacecraft. 
developed for the hypersonic separated flow jet interactions posed by this problem.

In addition to the basic aerodynamics of the Orion spacecraft, Orion designers must also analyze the performance of the Orion Launch Abort Vehicle (LAV), shown in Figure 20. The LAV is designed to provide an emergency crew escape capability in the event of a launch vehicle failure. It must be able to be operated anywhere in the flight trajectory from sitting stationary on the launch pad until shortly after first stage burnout. The vehicle is statically unstable and requires an active flight control system to maintain vehicle orientation throughout its flight. The LAV is powered by a main Abort Motor (AM) exhausting from the four large nozzles about midway up the forward tower. At the forward end of vehicle is the Attitude Control Motor (ACM), which is a single solid rocket motor that exhausts through eight nozzles arranged circumferentially around the top of the tower. Each of these eight nozzles can be metered individually to actively control the pitch and yaw attitude of the LAV.

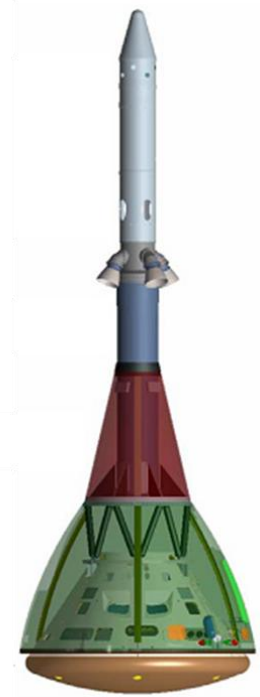

Figure 20. Orion Launch Abort Vehicle (LAV).

This control system and arrangement of nozzles has proven to be exceptionally difficult to analyze and predict vehicle performance. When both the AM and ACM are operating together, the ACM plumes can interact with the AM plumes as shown in Figure 21. The case depicted by the figure shows all eight ACM plumes firing simultaneously, but in reality, the ACM nozzles can be metered so that individual or select groups of nozzles can be operating while the others are effectively shut off. In these cases, the ACM jets can push on the AM plumes that then push on the back of the LAV to generate pitching or yawing moments on the vehicle in addition to simply the reactive moments due to the thrust of the individual ACM nozzles. These interactions are highly nonlinear and dependent on the flight conditions, and are particularly severe in the

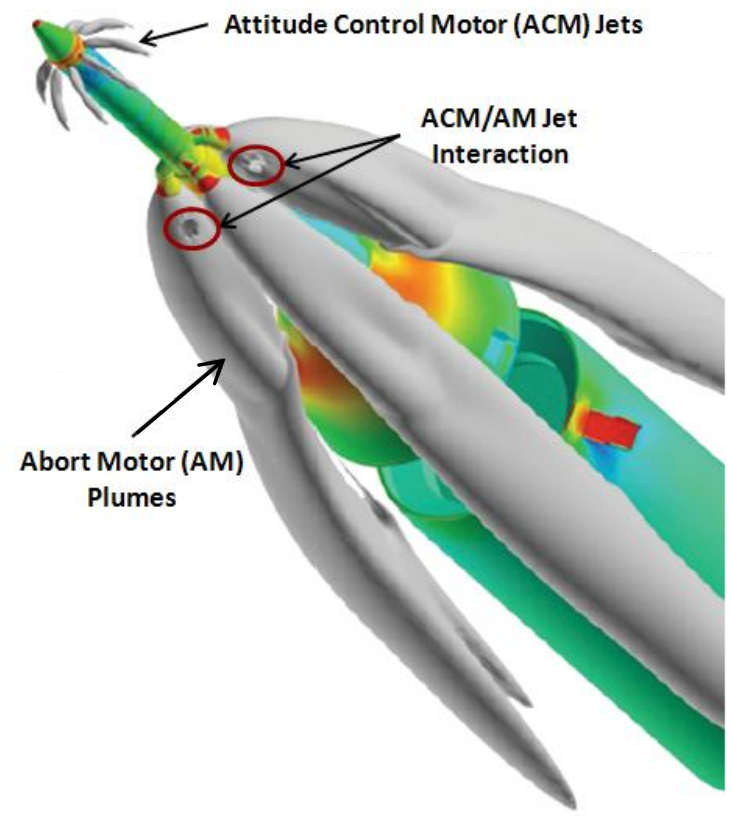

Figure 21. Orion LAV AM/ACM interaction.

transonic flight regime. The application of CFD to this flow is problematic due to the lack of experience and data for these types of interactions. The problem is compounded by the fact that wind tunnel testing of this type of interaction is very difficult, complicated, and expensive and in the end, it is virtually impossible to match flight conditions for this type of problem in a subscale wind tunnel test. CFD is the engineer's best tool to accurately predict these flows under full-scale flight conditions, but with the lack of data and experience in this problem, the uncertainties surrounding the CFD analyses are large.

An example of how this issue is challenging the Orion LAV developers is presented in Figure 22. This figure shows the CFD predicted flowfield for the Orion LAV with the main abort motors running and two of the ACM nozzles firing downward. At certain conditions in the transonic flight regime, the two downward-firing ACM jets become left-right asymmetric, even though geometrically and mathematically they should remain symmetric. This predicted asymmetry greatly reduces the pitch effectiveness of the ACM and produces a yawing moment on the vehicle when a pure pitch is intended from this nozzle firing combination. Depending on how the CFD method is initiated and executed, the asymmetry can be generated to the right or left, and in some cases the flow can remain symmetric.

Code-to-code comparisons show that this asymmetry is predicted consistently by all the CFD methods applied to the problem to date. Engineers are struggling with how to account for this phenomenon in the vehicle design. There are questions whether the phenomenon will ever surface during normal 


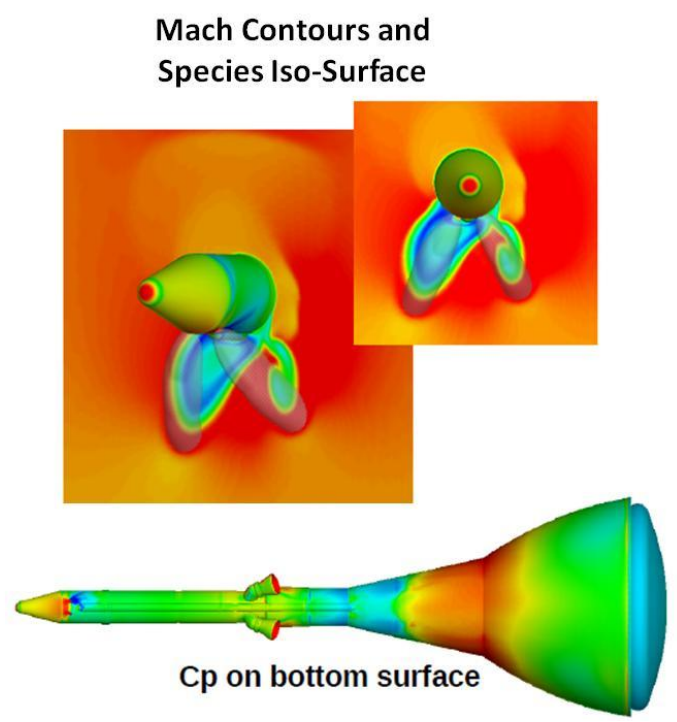

Figure 22. Orion LAV flow asymmetry predicted by CFD.

operations given the constantly changing flow conditions and ACM operating parameters. Even if it does occur during flight, the question becomes whether it will persist long enough to adversely affect the LAV performance.

Issues like this are having a detrimental impact on the vehicle designs as large performance margins must be built into the concepts with consequential penalties in weight and system complexity. Unlike the design and development of NASA's last manned spacecraft, the Space Shuttle, CxP is relying heavily on CFD to make design decisions and predict vehicle performance. The program is performing considerably less testing than during the Apollo and Space Shuttle programs and as a result we see engineers again applying large modeling uncertainty factors on analysis data that impacts vehicle designs. Testing that is being performed is serving the dual role of vehicle performance evaluation and method validation, but there simply isn't enough testing available to adequately validate and calibrate the wide range of CFD applications required for this program. In the case of the aforementioned ACM asymmetry, it is not clear that the phenomenon could be accurately predicted or characterized in the wind tunnel. So again, NASA is highly motivated to use the flight tests they have scheduled in support of the program to also acquire data that can be used to reduce the modeling uncertainty carried in the development of the vehicle.

In the last year, $\mathrm{CxP}$ has flown three test flights, the Max Launch Abort System, the Ares I-X demonstration flight and the Pad Abort 1 (PA-1) launch pad abort flight. Given the broad use of CFD and other analytical methods and the large modeling uncertainties associated with these analyses, each of these vehicles have been liberally instrumented with aerodynamic and aerothermodynamic sensors to capture steady pressure data, buffet pressure data, aeroacoustic pressures, and heating data, as well as flight telemetry information from which flight conditions and integrated force and moment data can be derived. So NASA CFD application engineers and development researchers suddenly have new sources of flight data against which to evaluate their capability and methods. Flight data can be extremely difficult and rare to acquire in the normal develop, validate, apply CFD maturation cycle. But through the liberal application of our CFD capability, particularly for cases where little or no validation data or past experience is available, developers can suddenly find themselves in a situation where managers are highly motivated to provide ground test and flight data that can be used to help validate their methods and reduce uncertainties.

In some areas, engineers and researchers have begun to identify Flights-of-Opportunity where existing flight projects are evaluated and targeted for their potential to produce needed flight data for methodology validation. As a result of this type of forward thinking, boundary layer transition experiments have recently been performed on the Space Shuttle ${ }^{8,9}$ as it reenters the atmosphere. Instrumentation has also been added to the flight manifest of the Mars Science Laboratory entry vehicle to measure heatshield performance parameters as it enter the Martian atmosphere ${ }^{10}$.

\section{REQUIREMENTS, PROS, AND CONS OF THE "DEVELOP, APPLY, VALIDATE" CFD MATURATION STRATEGY}

When the validate and apply processes begin to merge and switch, engineers and researchers who have been classically known as CFD developers must begin to work more closely with those applying the CFD methods. In the classical paradigm, a developer took his methodology all the way through the validation stage and when complete, delivered an analysis capability that had been tested for a specific range of problems. Those applying the methods knew this range of applicability and had confidence, and proof, of the methods the ability to predict these specific problem classes. When the codes begin to be applied outside their known range of validation, engineers look to the people most experienced with the method, the developers, to help guide them through the application. Suddenly developers find themselves answering difficult questions about specific problems pertaining to specific vehicle designs.

As a result of this closer interaction, the developer finds themselves performing tasks differently than in the past. First, they find themselves analyzing and understanding more cases than the handful of validation cases tested under the classical strategy. Vehicle applications typically involve parametric analyses of hundreds if not thousands of cases, a handful or range of which might generate some poorly understood flow phenomenon or unanticipated 
performance. The application engineers thus look to the developers to help them determine if the methodology is indeed capable of predicting these events and if they are physically realistic or an artifice of the numerical methodology.

This forces developers to exercise cases on geometries that are often much more complex than the typical validation case. To sufficiently refine these geometries, the grids are also usually much larger than for the typical validation cases analyzed. For instance, it is not unusual for grids on the Ares I or Orion LAV to exceed 50 million grid points.

Analyzing data on these more complicated geometries and larger grids put a greater burden on post processing and interpretation of the data. The physical characteristics of typical CFD validation cases are often very well known by the development community and it is usually apparent whether a given methodology is behaving reasonably. This is not the case when the applications begin to outpace the validation. Physical characteristics of these applications are usually not very well understood and it often takes a large amount of post processing to cast results in a form that best highlights these characteristics.

Finally, as the physical characteristics and the performance of the methodology become better understood, opportunities for ground and flight testing begin to emerge. These tests are typically focused on predicting the performance and reducing uncertainty of a vehicle or system, but when analysis uncertainties are high, they also become opportunities to validate the methods used to predict performance. Again, the application engineers look to the developers to help them define and place instrumentation so as to best capture the physical events that are challenging the methods. As a result, the developer can also find themselves becoming more involved in the tests of the project, as opposed to tests formulated specifically to validate methodology.

In the "Develop, Apply, Validate" strategy, the developer must become more aligned with the application engineers and their problems. There are definite pros and cons to this alignment. Many of the pros have been highlighted previously in this paper, the most prevalent of which is that the developers become better aware of the problems their methods are being asked to analyze and they receive direct input as to the problems that require address for future development. Access to validation tests can also be improved. Acquiring sufficient test data is always a struggle under the classical "Develop, Validate, Apply" strategy; acquiring flight data under this paradigm is very rare. Also, the methods get to the applications engineers faster because they don't go through the validation process before they begin application.

Many of the cons are fairly obvious. In the extreme, methods could be introduced to the engineering community before they have undergone even the most basic of validation. At best, applying methods outside their known validation range is higher risk and the associated large uncertainties associated with this strategy are warranted. The full development cycle for methodology is slower using this strategy since significant application of the method may have occurred prior to the acquisition of sufficient validation data to certify the methodology. The development cycle can also become a more organic process with it becoming increasingly difficult to define the beginning or the end of the cycle. This could be seen as either a pro or a con, especially when one considers the effort required in advocating and initiating a new development campaign; a selfsustaining process can be very attractive.

\section{SUMMARY}

NASA is experiencing an explosive growth in CFD applications that is outpacing the validation of their methods. Two large reasons for this growth are the increased technical insight into Space Shuttle operations as precipitated by the Columbia accident and the development of new manned spacecraft under the Constellation Program. Under these programs engineers are applying CFD to problems that were not or could not be foreseen in the standard development/validation cycle. The high-priority, time-critical nature of these analyses do not provide sufficient opportunity to validate the methods prior to their application. Application of the methods in these environments lead to large uncertainties and design margins being applied to the vehicles and systems to offset the lack of knowledge and experience in the specific application of the CFD methodology. These margins can have a significant impact on vehicle performance and reducing uncertainty becomes a critical issue to vehicle operations and designs. To reduce uncertainties, ground and flight testing is formulated and CFD developers suddenly find themselves in a position to acquire the much-needed data to validate their methods. Thus while there are definite costs to operating in a "Develop, Apply, Validate" strategy, there are ancillary benefits as well.

NASA has naturally evolved into this strategy as a result of mission events and mission requirements. It is unlikely that anyone would have adopted this approach as an a priori strategy, simply because it does not fit the well-established logical and systematic approach to method development. But now that the Agency has refined and exercised this strategy for several years and started to realize some of its benefits, it warrants a closer look as an alternative approach to method development. It can be particularly attractive in environments where advocacy for tool development can be a challenge. 


\section{REFERENCES}

1. Gehman, Jr., H. W., et al., "Columbia Accident Investigation Board, Report Volume 1,” National Aeronautics and Space Administration, August, 2003.

2. Buning, P. G., et al., "Numerical Simulation of the Integrated Space Shuttle Vehicle in Ascent," AIAA Paper 88-4359-CP, August, 1988.

3. Pearce, D. G., et al., "Development of a Large Scale Chimera Grid System for the Space Shuttle Launch Vehicle," AIAA Paper 93-0533, January, 1993.

4. Gomez, R. J. and Ma, E. C., "Validation of a Large Scale Chimera Grid System for the Space Shuttle Launch Vehicle," AIAA Paper 94-1859, June, 1994.

5. Gomez, R. J., et al., "STS-107 Ascent CFD Support," AIAA Paper 2004-2226, July 2004.

6. Murman, S. M., Aftosmis, M. J., and Rogers, S. E., "Characterization of Space Shuttle Ascent Debris Aerodynamics Using CFD Methods, “AIAA Paper 2005-1223, January, 2005.

7. Eby, M. A., "Aerodynamics Module for the Space Shuttle Foam Debris Probabilistic Risk Assessment," AIAA Paper 2008-6909, August, 2008.

8. Anderson, B. A., et al., "Boundary Layer Transition Flight Experiment Overview and In-Situ Measurements," AIAA Paper 2010-0420, January, 2010.

9. Horvath, T. J., et al., "The HYTHIRM Project: Flight Thermography of the Space Shuttle during Hypersonic Re-entry," AIAA Paper 2010-0241, January, 2010.

10. Gazarik, M. J., et al., "Overview of the MEDLI Project,” IEEEAC Paper \#510, March, 2008. 